\title{
Neue Weiterbildungsmöglichkeit zum Thema Organspende
}

Isabelle Not ${ }^{a}$, Luca Martinollib, Franz Immerc, Comité national du don d'organes CNDO/Swisstransplant*, Fachausschuss Schulungen des CNDO/Swisstransplant**

a Dr. med. vet., Swisstransplant, Schweizerische nationale Stiftung für Organspende und Transplantation; ${ }^{b}$ Dr. med., CMO Swisstransplant; c PD Dr. med., CEO Swisstransplant

Eine repräsentative Umfrage zeigt: Der Hausarzt ist bevorzugter Ansprechpartner bei Fragen zur Organspende - entsprechendes Fachwissen wird vorausgesetzt. Um dieses zu vermitteln, wurde eine neue Lernplattform erstellt. Ursprünglich für Fachpersonal in den Spitälern entwickelt, ist sie durch modularen Aufbau und die Möglichkeit zum Selbststudium auch für Spezialärzte und/oder Grundversorger ein idealer Einstieg in die komplexen Abläufe des Organspendeprozesses.

\footnotetext{
* Mitglieder CNDO (Comité national du don d'organes, nationaler Ausschuss für Organspende): PD Dr. med. Markus Béchir, Schweizer Paraplegiker-Zentrum, Nottwil; PD Dr. med. Yvan Gasche, HUG, Genf; **Petra Bischoff, Inselspital, Bern; ${ }^{* *}$ Corinne Delalay-Marti, Hôpital du Valais, Sion; Dr. med. Philippe Eckert, CHUV, Lausanne; Dr. med. Susann Endermann, Kantonsspital St. Gallen St.Gallen; Eva Ghanfili, Ospedale Civico, Lugano; Prof. Dr. med. Christoph Haberthür, Klinik Hirslanden, Zürich; PD Dr. med. Franz Immer, Swisstransplant, Bern; Dr. med. Renato Lenherr, USZ, Zürich; **Dr. med. Sven Mädler, Pallas Kliniken, Olten; Diane Moretti, HUG, Genf; **Stefan Regenscheit, USZ, Zürich; Dr. med. Thomas Riedel, Inselspital, Bern; Dr. med. Thomas Sauter, Inselspital, Bern; Dr. med. Kai Tisljar, Universitätsspital Basel, Basel; Dr. med. Jan Wiegand, Lindenhofspital, Bern

** Mitglieder Fachausschuss Schulungen:

Dr. med. Roger Lussmann, Klinik Hirslanden, Zürich. Die weiteren Mitglieder des Fachausschusses Schulungen sind in der Mitgliederliste CNDO mit ${ }^{* *}$ markiert.
}

Nouvelle offre de formation sur le don d'organes La Fondation nationale suisse pour le don et la transplantation d'organes, Swisstransplant, et son Comité national du don d'organes (CNDO) ont mis sur pied un nouveau programme de formation standardisé destiné au personnel médical spécialisé directement impliqué dans le processus du don d'organes. Disponible en trois langues (fr/de/it), cette formation mixte comprend dix modules à distance et deux cours en présence sur la communication et la médecine.

Les personnes intéressées, par exemple médecins de famille ou médecins spécialistes qui ne sont pas directement impliqués dans le don d'organes, peuvent suivre les modules qu'ils désirent après avoir effectué le module de base consacré aux connaissances fondamentales dans le domaine du don d'organes en Suisse.

Des crédits de formation pour les différents modules et cours en présence ont été demandés auprès des sociétés de discipline médicale. La formation se conclut par un examen qui débouche sur le certificat d'«Expert suisse pour le don d'organes CNDO/SWT». De plus amples informations, y compris le formulaire d'inscription, sont disponibles sur le site de Swisstransplant.
Das Thema Organspende ist im Spital und in den Praxen ein seltenes aber dennoch sehr relevantes Thema. Bei einer Zahl von 14,4 postmortalen Spendern pro Million Einwohnern, was 117 Patienten im Jahr 2014 entspricht, ist dies verständlich. Swisstransplant und die Intensiv- und Notfallmediziner im nationalen Ausschuss für Organspende CNDO haben schon länger erkannt, dass es für die Kollegen in Praxis und Spital schwierig ist, die Entwicklungen und Neuerungen auf dem Gebiet der Organspende und Transplantation zu überblicken und bei Fragen kompetent Auskunft zu geben. Wussten Sie zum Beispiel, dass das Alter der Organspender in der Schweiz eine Spanne von wenigen Monaten bis 88 Jahren umfasst und deren Durchschnitt 52 Jahre beträgt? Oder, dass rund ein Drittel der Spender vorgängig reanimiert wurde? Eine repräsentative Umfrage vom Frühjahr 2015 zeigt ausserdem, dass der Hausarzt der bevorzugte Ansprechpartner bei Fragen zur Organspende ist (Abb. 1).

\section{Auch die Hausarztpraxis braucht Fach- wissen zum Thema Organspende}

Aus diesem Grund haben Swisstransplant (SWT) und das CNDO vor zwei Jahren mit der Entwicklung eines modularen Lehrgangs begonnen, der die vielen Aspekte und unterschiedlichen Bereiche des Organspendeprozesses (OSP) kompakt abbildet und eine einheitliche Informationsgrundlage schafft. Gleichzeitig erfüllt dieser Lehrgang auch die Anforderungen des BAG-Aktionsplans "Mehr Organe für Transplantationen", der zum Ziel hat, per Ende 2018 die postmortale Spende- 


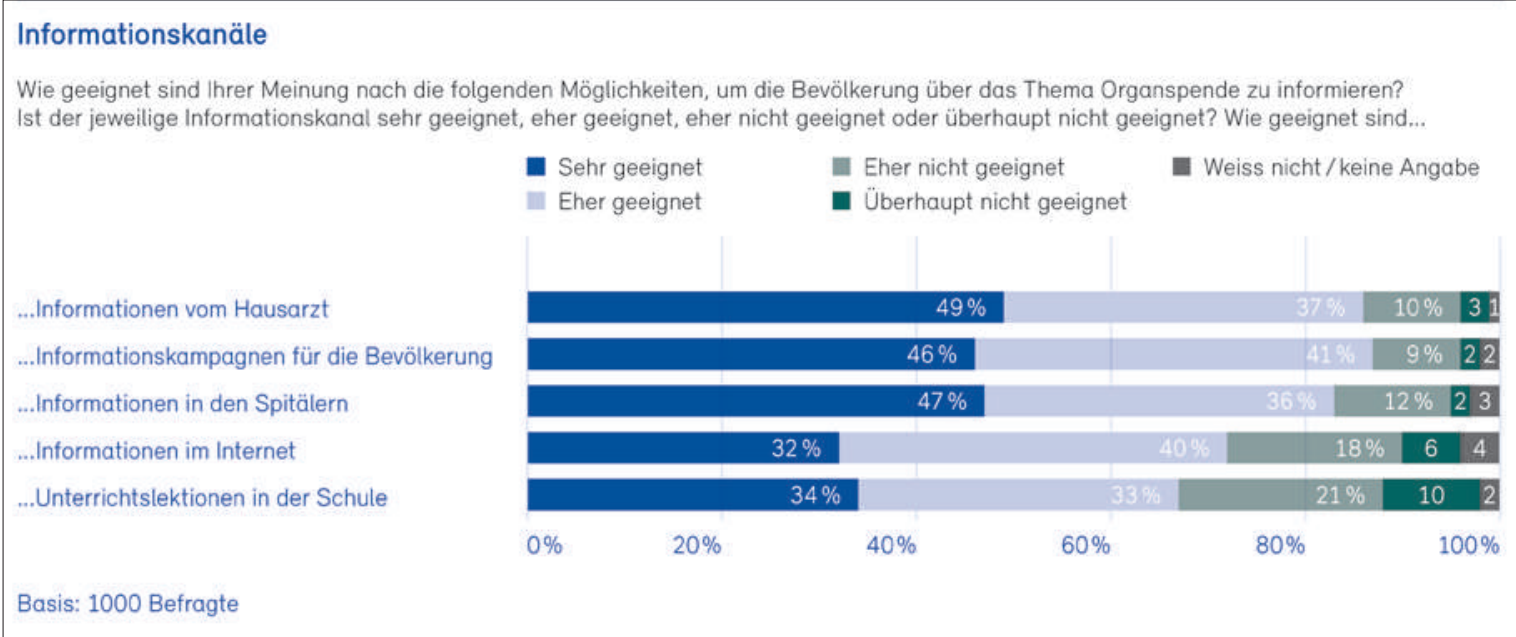

Abbildung 1: Resultate aus der DemoSCOPE-Umfrage unter 1000 Schweizern im Frühjahr 2015.

rate auf 20 Spender pro Million Einwohner zu erhöhen. Swisstransplant wurde in diesem Rahmen vom Bund die Aufgabe zugewiesen, einen nationalen Lehrgang für das im OSP tätige, medizinische Fachpersonal vom Rettungssanitäter über die Kollegen in den Arztpraxen bis hin zum Fachpersonal in den Spitälern umzusetzen.

\section{Aufbau und Inhalt des Blended Learning Organspendeprozess (OSP)}

Aufgrund der hohen beruflichen Belastung müssen Weiterbildungen im medizinischen Bereich besonders vielen Anforderungen genügen. So auch diejenige zum OSP: Die Inhalte sind umfangreich und komplex, erfordern einen hohen Aktualitätsgrad, eine laufende Qua- litätskontrolle und sollen in der regionalen Landessprache verfügbar sein. Ort, Zeit und Dauer der Ausbildung müssen flexibel sein. Die Absolventen sollen in ihrem eigenen Tempo lernen und die Schwerpunkte ihres Trainings selber festlegen können. Ausserdem sollen der Austausch untereinander gefördert und schliesslich auch die praktischen Fähigkeiten trainiert werden. Diese vielseitigen Anforderungen erfüllt ein Blended Learning zurzeit am besten: Dabei werden die benötigten Inhalte auf einer digitalen Lernplattform zur Verfügung gestellt und mit Präsenzkursen ergänzt. Der neue Lehrgang OSP kombiniert zehn Module online-basiertes Selbststudium mit zwei Präsenzkursen zu den Schwerpunkten «Medizin» und «Kommunikation» (Abb. 2).

\section{Schweizerischer Experte im Organspendeprozess CNDO/SWT}

Präsenzkurs

Aufbaumodule

Kommunikation

Präsenzkurs

Aufbaumodule

Medizin und

Qualität

Basismodul
Kommunikation

\begin{tabular}{|c|c|c|c|}
\hline $\begin{array}{l}\text { Kommunikation } \\
\text { im medizinischen } \\
\text { Umfeld }\end{array}$ & $\begin{array}{l}\text { Kommunikation } \\
\text { Breaking Bad } \\
\text { News }\end{array}$ & $\begin{array}{l}\text { Kommunikation } \\
\text { Organspende- } \\
\text { prozess (Teil 1) }\end{array}$ & $\begin{array}{l}\text { Kommunikation } \\
\text { Organspende- } \\
\text { prozess (Teil 2) }\end{array}$ \\
\hline
\end{tabular}

Medizin und Qualität

\begin{tabular}{l|l|l|l|l|}
$\begin{array}{l}\text { Spender- } \\
\text { erkennung }\end{array}$ & $\begin{array}{l}\text { Hirntod- } \\
\text { diagnostik }\end{array}$ & $\begin{array}{l}\text { Spender- } \\
\text { behandlung }\end{array}$ & $\begin{array}{l}\text { Organ- } \\
\text { entnahme }\end{array}$ & $\begin{array}{l}\text { Prozesse/ } \\
\text { Qualität }\end{array}$ \\
\hline Grundwissen zur Organspende in der Schweiz
\end{tabular}

Abbildung 2: Aufbau des Blended Learning zum Organspendeprozess. 


\section{Der Lehrgang richtet sich an unterschied- liche Zielgruppen}

Zielgruppen ohne direkte Tätigkeiten im Organspendeprozess, z.B. medizinische Grundversorger und/oder Spezialärzte, können nach dem obligatorischen Basismodul weitere Module nach Wahl bearbeiten und sind nicht verpflichtet, die Präsenzkurse zu besuchen. Das Basismodul enthält grundlegendes Wissen zum Organspendewesen in der Schweiz, das jedem Arzt geläufig sein sollte. Dazu gehören die gesetzlichen Grundlagen, die Organisation der Spendenetzwerke, die Kontraindikationen für Organspender und die sechs Transplantationszentren mit ihren aktuellen Programmen.

Im gültigen Vertrag zum Aktionsplan haben das BAG, die Stiftung Swisstransplant und das CNDO gemeinsam als bindende Zielsetzungen vereinbart, dass bis Ende 2016 80\% der lokalen Koordinatoren das Basismodul absolviert haben. Lokale Koordinatoren sind Ärzte und Pflegefachpersonen auf Intensiv- und/oder Notfallstationen, die eine aktive Funktion im OSP haben und die gemäss gesetzlicher Grundlage durch die Spitäler, bzw. die Kantone, der Nationalen Zuteilungsstelle Swisstransplant gemeldet werden müssen. Bis Ende 2017 sollen 60\% der Lokalen Koordinatoren das ganze Blended Learning mit dem Zertifikat «Schweizerischer Experte Organspendeprozess CNDO/SWT» abgeschlossen haben. Daher hat das CNDO für diese Mitarbeiter die Teilnahme für obligatorisch erklärt.

\section{Kontrollierte Lernfortschritte, Lernziele, integrierte Fragen und Abschlusstests}

Am Anfang jedes Moduls werden Lernziele definiert und automatisiert ausgewertete Online-Tests geben eine unmittelbare Rückmeldung über den individuellen Lernfortschritt. Um sicherzustellen, dass alle Teilnehmer bei den ergänzenden Präsenzveranstaltungen auf einem vergleichbaren Wissensstand sind, müssen bei diesen Tests $80 \%$ der möglichen Punktzahl erreicht werden. Für jedes erfolgreich absolvierte Modul wird eine persönliche Teilnahmebestätigung ausgestellt. Das in den zehn Modulen erworbene theoretische Wissen wird anschliessend in den beiden Präsenzveranstaltungen in die Praxis umgesetzt. Voraussetzung für die Teilnahme am Präsenzkurs "Medizin» sind die fünf medizinischen Module «Spendererkennung», «Hirntoddiagnostik», «Spenderbehandlung», «Organentnahme» und «Prozesse/Qualität». Für den Präsenzkurs «Kommunikation» müssen vorher die vier Module "Kommunikation im medizinischen Umfeld", «Überbringen schlechter Nachrichten» und «schwie- rige Gespräche im Organspendeprozess» (Teile 1 und 2) bearbeitet werden.

\section{Präsenzkurse}

Das neue Blended Learning integriert die bereits seit fünf Jahren von Swisstransplant angebotenen, sehr erfolgreichen zweitägigen Kommunikationskurse für Ärzte und Pflegefachpersonen (auch ausserhalb des OSP). Da sich die theoretischen Inhalte in den vier neuen Kommunikationsmodulen wiederfinden, wurden sie durch einen eintägigen Kurs ersetzt. An diesem Tag liegt der Schwerpunkt auf der Gesprächssimulation mit ausgebildeten Schauspielern. Je nach Drehbuch übernehmen diese in den Gesprächen die Rolle von Arzt, Pflegefachperson oder Angehörigen, um so den Teilnehmern das eigene Verhalten im Kontext dieser Situation praxisnah aufzuzeigen und auch mögliche Varianten durchzuspielen. Der Kurs wird von speziell ausgebildeten Psychologen geleitet, die Teilnehmerzahl ist auf 14 beschränkt, um allen eine aktive Mitarbeit zu ermöglichen.

Im Gegensatz zum Präsenzkurs «Kommunikation» wird der medizinische Präsenzkurs in zwei Versionen angeboten: Einer richtet sich an Pflegefachpersonen, der andere an Fachärzte Intensivmedizin und Kollegen in Ausbildung zum Facharzt Intensivmedizin. Diese zweitägigen Kurse vertiefen die Kenntnisse aus den Modulen «Spendererkennung», «Hirntoddiagnostik» und «Spenderbehandlung» mit Kurzvorträgen, Gruppenarbeiten, Erfahrungsaustausch und vor allem Fallsimulationen.

\section{Credits medizinischer Fachgesellschaften und Zertifizierung}

Die Weiterbildung nimmt, je nach Vorkenntnissen, zwei bis vier Stunden pro Modul in Anspruch. Entsprechend den Kriterien der verschiedenen medizinischen Fachgesellschaften werden Weiterbildungs-Credits für die einzelnen Module und separat für die Präsenzkurse vergeben. Bisher werden von einigen Fachgesellschaften für die zehn E-Learning-Module maximal 29 Credits vergeben.

Um das Zertifikat zu erlangen, ist die Abfolge der Bearbeitung der Module vorgegeben. Erst nach der erfolgreichen Absolvierung des Basismoduls können die fünf Module mit medizinischen Schwerpunkten absolviert werden. Daran anschliessend folgen die vier Module, die der Kommunikation gewidmet sind. Für die Zertifizierung zum «Schweizerischen Experten Organspendeprozess CNDO/SWT» wird zusätzlich ein Abschlusstest über die Inhalte aller Module durchgeführt. 


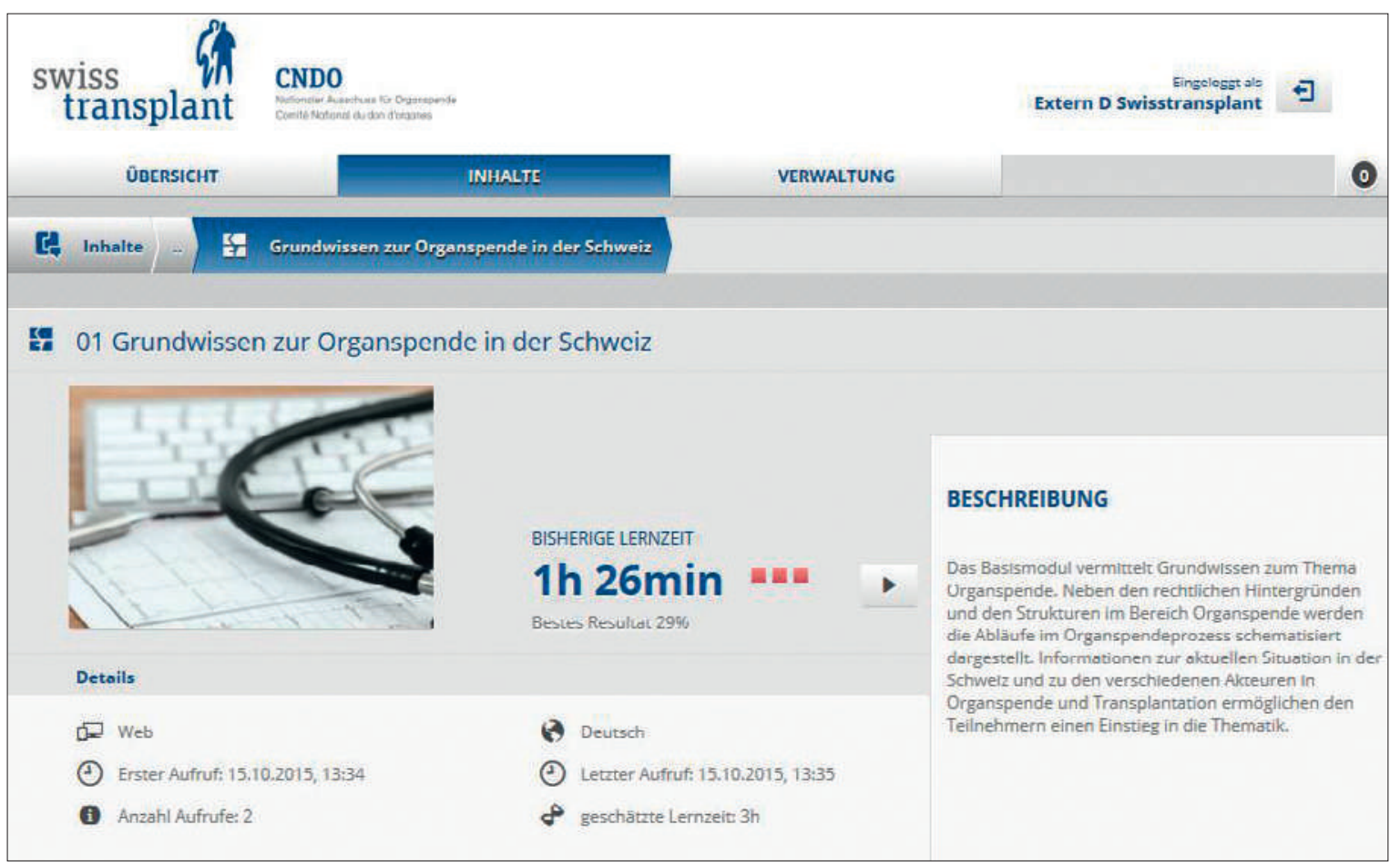

Abbildung 3: Webansicht der Lernplattform.

Korrespondenz: PD Dr. med. Franz Immer Swisstransplant Effingerstrasse 1 Postfach CH-3011 Bern Tel. 0581238002 franz.immer[at] swisstransplant.org

\section{Geglückter Start mit Qualitätsprüfung und Testlauf in der Praxis}

Im Sommer hat eine Pilotgruppe von 20 Intensivmedizinern und Intensivpflegefachpersonen aus der Deutschschweiz, der Romandie und dem Tessin die zehn Module gründlich geprüft. Die Lernplattform wurde mit diesen Rückmeldungen aus der Praxis überarbeitet und am 1. Oktober 2015 für die Teilnehmer zugänglich gemacht. Das Blended Learning ist mit Absicht nicht für die Arbeit mit mobilen Geräten wie iPad, Smartphone etc. ausgelegt, da die einzelnen Module klinische Bilder und Videos enthalten, die nicht ausserhalb des medizinischen Umfeldes verbreitet werden sollen. Der Datenschutz der gezeigten Fälle bleibt dabei selbstverständlich gewährleistet.

\section{Zugang und technische Voraussetzungen}

Interessenten, die ihr Wissen über die Organspende erweitern wollen, registrieren sich direkt auf der Webseite von Swisstransplant unter www.swisstransplant. org/blendedlearning oder schreiben eine Anfrage an cndoblended.learning[at]swisstransplant.org. Jeder Teilnehmer erhält ein eigenes Passwort. Der persönliche Zugang zur Lernplattform (Abb. 3) bleibt nach der Eröffnung vier Monate bestehen. Genauere Beschreibungen der einzelnen Module sowie freie Termine für die Teilnahme an den Präsenzkursen finden sich ebenfalls auf der Website von Swisstransplant. 